\title{
Research on Carbon Fiber Reinforced Concrete Beam Bridge Based on Non- probability Theory
}

\author{
Xuyong CHEN \\ School of Resource and Civil Engineering \\ Wuhan Institute of Technology \\ Wuhan, China \\ E-mail: cxy1314k1@126.com
}

\author{
Lu LUO \\ School of Resource and Civil Engineering \\ Wuhan Institute of Technology \\ Wuhan, China \\ E-mail: 1025440100@qq.com
}

\author{
Tengteng SONG \\ School of Resource and Civil Engineering \\ Wuhan Institute of Technology \\ Wuhan, China \\ E-mail: 2447340414@qq.com
}

\begin{abstract}
The non-probabilistic reliability theory is introduced to assess bridges which are in active service. A model of simply supported girder bridge is built based on this theory. Theoretical method and failure point optimization method based on the finite element software are used respectively for computation prior to strengthen of the girder bridge model to validate the effectiveness of failure point optimization method. In the meantime, reliability evaluation is conducted. After being strengthened with carbon fiber cloth, the model is implemented with non-probabilistic reliability evaluation. Afterwards, the evaluation is further conducted under different conditions by means of altering the width and the layer of carbon fiber cloth respectively. And afterwards, an optimal carbon-fiber-reinforced plan is worked out.
\end{abstract}

Keyword-non-probabilistic reliability; arch bridge; failure point optimization method; evaluation; strengthened by CFRP

\section{INTRODUCTION}

As an important part of road, bridge plays an important role in traffic safety and smoothness. With the development of social economic development, there are a very large number of bridges. At the present, there are 760 thousands bridges with a total length of more than $40000 \mathrm{~km}$ all around China. However, the problem of bridge disease has become increasingly serious. Diseases and defects in different levels of current bridges seriously harm the normal use of the bridge. China has entered a period when new construction and maintenance are paid equal importance. The sticking carbon fiber reinforcement method, which has been widely applied to structure maintenance and reinforcement, has high construction efficiency, reinforcement effectiveness and comprehensive benefits, with characteristics of light weight, high strength and outstanding design-ability. Due to the complexity of reinforcement components and difference in reinforcement position, it is essential to research the reliability of reinforcing components[1]-[8]. Current studies on the reliability of carbon fiber reinforcing bridges are mainly confined to the probability theory as the base.
Although the research based on probabilistic reliability has achieved fruitful results, but it still has many shortcomings. As the probabilistic reliability is sensitive to the probability, a tiny error in probability data may lead to big error in the calculation result of structure reliability [2]. At the same time, it is difficult to get large enough data which meet the requirement of probability methods, causing there are many problems in probabilistic reliability research. The nonprobabilistic reliability theory is also widely used in engineering studies, which needs relatively less data and is easy to be researched with accurate results.

Non-probabilistic reliability theory is becoming matured day by day. The concept, non-probabilistic reliability, has been put forward on the basis of researches and analysis of the interval model, that is, the volume ratio of the structural security domain and the interval variable domain is defined as the structural reliability measurement. The nonprobabilistic reliability analysis of the interval theory does not need specific distribution of parametric variables. It only needs to determine the upper and lower limits of parameters, effectively reducing the computational complexity with better adaptability. Jiang Tao [10] has put forward to a onedimension optimization algorithm to calculate nonprobabilistic reliability index. It transforms the solution of non-probabilistic index into the rooting of single-variable equation. The one-dimensional optimization algorithm in the literature transforms the interval variable to standard interval variable, obtains the limit state equation about failure and determines reliable index of the monotonicity calculation of the objective function[11]. Chen Xuyong[12] first introduces the non-probabilistic theory into the evaluation of existing bridges and evaluates the non-probabilistic reliability of a simple simply-supported beam bridge. There is still a lot of specific work to be studied on the non-probabilistic reliability evaluation of existing bridges. This article studies the digital model of the non-probability theory's reliability assessment on the simply-supported beam bridge based on the non-probabilistic theory and calculates the reliability of the proposed method. 


\section{The Non-Probabilistic RELIABLITY OF THE SIMPLY-SUPPORTED BEAM BRIDGE}

\section{A. Non-probabilistic Reliability Theoretical Model}

The non-probabilistic reliability model of the calculated structure is obtained according to the interval analysis method. Assume the calculated interval variable set associated with the structure, $x=\left\{x_{1}, x_{2}, \ldots, x_{n}\right\}$, among which $x_{i} \in X_{i}(i=0,1, \ldots, n)$. Then the function in the limit state can be calculated as:

$$
M=f(x)=f\left(x_{1}, x_{2}, \ldots, x_{n}\right)
$$

When $f(x)$ is the continuous function of $x_{i}(i=0,1, \ldots, n)$ and the function $M$ is an interval variable, its mean and deviation can be calculated, which are separately expressed as $M^{c}$ and $M^{r}$. Set

$$
\eta=\frac{M^{c}}{M^{r}} \in\left\{\begin{array}{cc}
{[1,+\infty)} & \text { reliableandsecure } \\
(-1,1) & \text { unreliable } \\
(-\infty,-1] & \text { fail }
\end{array}\right.
$$

In terms of engineering practice, when $-1<\eta<1$, the structure is regarded unreliable; when $\eta>1$, the structure is regarded reliable; the larger the value is, the more structure is. The structural security can be determined according to the size between $\eta$ and 1 . In terms of geometric meaning, the extended interval variables are regarded as standard interval variables and the structural reliability can be expressed by the distance of the shortest path between the ordinate origin and the function failure plane. Fig. 1 and 2 respectively give normalized interval variables after expansion and measure the structural reliability by the distance of the shortest path between the origin and the structural function. When $\eta>1$, the actual fluctuation range and the failure range of the structural function have no public intersection, indicating that the structure is secure at that time. The larger value indicates the more secure and reliable structure.

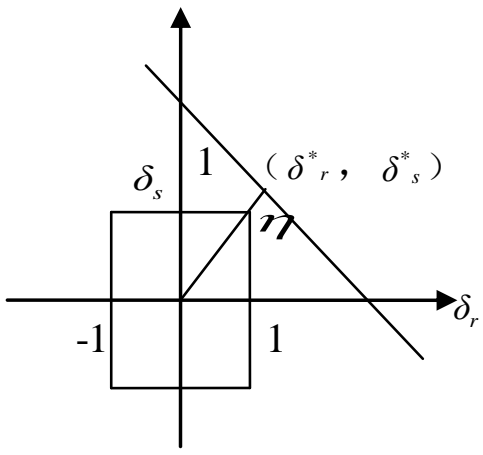

Figure 1. Linear performance function

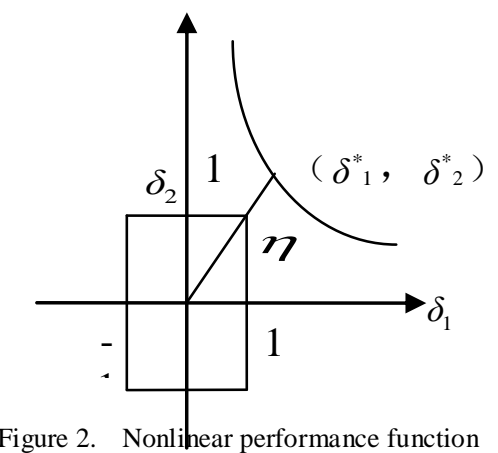

\section{B. Sensitivity Analysis of Parameters}

The bridge structure is sensitive to some factors. When these factors change a little, the bridge's carrying capacity changes a lot; but it responds slowly to some other factors. As different factors play different role in the bridge structure, we can analyze the sensitivity of these factors before collecting data to preliminary identify the sensitivity of each factor. For factors with high sensitivity, we can collect data as much as possible when detecting the bridge to make data more accurately simulate the actual bridge; for factors with poor sensitivity, an initial rough range can meet the requirements of assessment and calculation.

The objective of sensitivity analysis is to identify the key parameter affecting the model performance which plays an important role in model parameterization and uncertainty calibration, optimization and quantification[13]. The operation is generally keeping the other parameters unchanged by changing only one input variable to determine the influence on the model result. Change the input variable and observe the change of calculated result. The sensitivity analysis can effectively determine which input variables have larger impact on the calculation of the model. In the actual project, this method can reduce the work of data collection and effectively improve the working efficiency.

The primary problem of the sensitivity analysis of the bridge is to determine reasonable function based on the failure criterion. The specific function of the simplysupported beam bridge can be calculated through the failure criterion of double reinforcement rectangle section. The nonprobabilistic reliability index is solved through the modified one-dimension optimization algorithm as follows:

1) Solving the function $N$

Through the reliability analysis of the simply-supported beam bridge, it is shown that its worst stress section is the mid-span and the section of the mid-span has the biggest bending moment. Its limit state equation is:

$$
N=R-S
$$

Among them $\mathrm{R}$ is the structural resistance, $\mathrm{S}$ is the loading effect. Calculate $\mathrm{R}$ according to the formula of the bearing capacity of the normal section of flexural members; the load effect $S$ can be calculated by analyzing the internal 
force of the bridge under the bridge's dead weight and the lane load. Its calculation expression is as follows:

$$
\begin{gathered}
R=f_{s d} A_{s}\left(h_{0}-a_{s}^{\prime}\right)-f_{c d} b x\left(x / 2-a_{s}^{\prime}\right)+f_{c d} b x\left(h_{0}-x / 2\right)+f_{s d}^{\prime} A_{s}^{\prime}\left(h_{0}-A_{s}^{\prime}\right) \\
S=0.125 \gamma b(h+d) l_{0}^{2}+0.25 \alpha P l_{0}
\end{gathered}
$$

Then the formula (3) can be expressed as follows:

$$
N=f_{s d} A_{s}\left(h_{0}-a_{s}^{\prime}\right)-f_{c d} b x\left(x / 2-a_{s}^{\prime}\right)-0.125 \gamma b(h+d) l_{0}^{2}-0.25 \alpha P l_{0}
$$

\section{2) Reliability assessment process}

Analyze the simply-supported beam bridge according to the improved one-dimensional optimization algorithm as follows:

A. obtain the parameters needed for calculation and analysis according to the measurement and study on field;

B. arrange and analyze measured data, represent each parameter in form of interval and calculate each variable's mid-value, deviation and dispersion;

C. obtain the expressions of the resistance and the internal force of the solid plate respectively to establish the limit function;

D. analyze the sensitivity of the function and calculate parameter factors of the function with high sensitivity and low sensitivity;

E. solve the non-probabilistic reliability through the improved one-dimensional optimization algorithm. Use the finite element software MATLAB to solve the equation of higher degree. When the non-probabilistic reliability index $^{-1<\eta<1}$, the structure is not reliable; when $\eta>1$, the structure is reliable. Judge the result and assess the reliability of the bridge security before reinforcement.

\section{ACTUAL ENGINEERING Reliablity ASSESSMENT AND BRIDGE REINFORCEMENT SCHEME}

\section{A. Project overview}

Sanmen Bridge is located on the Guangshui Songchang Road in Suizhou city with the center stake number of $\mathrm{K} 136+260$. It is a reinforced concrete girder bridge with a span composition of $1 \times 8 \mathrm{~m}$, total length of $24.1 \mathrm{~m}$, original bridge deck width of $12 \mathrm{~m}$, and is divided into two sections of the motor way and the sidewalk. The upper superstructure is the precast reinforced concrete solid plate and the substructure adapts the gravity-typed abutment. The bridge abutment is made of mortar rubble, with cone slope protection on both sides. The designed load grade of the whole bridge is Automobile -15. Due to its long operation period, it is designed according to the Road-II Load.

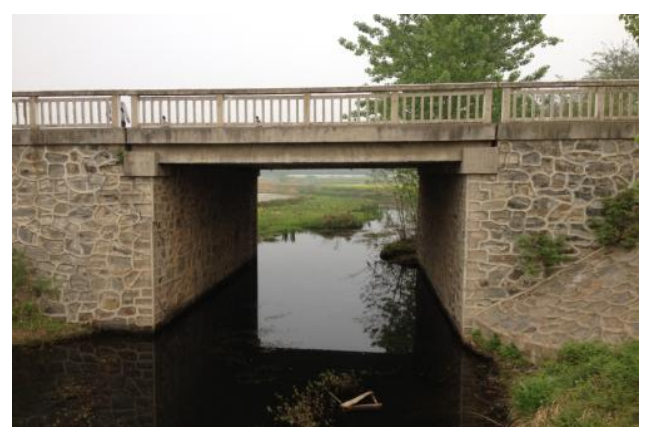

Figure 3. Old Bridge of structural map

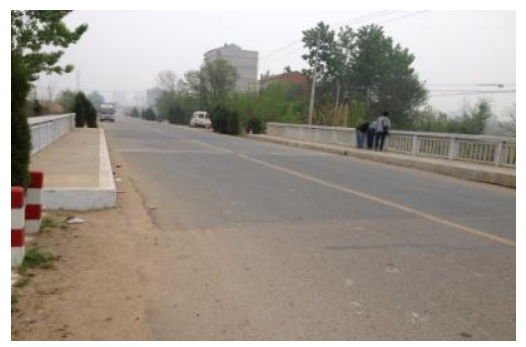

Figure 4. Upper structure construction diagram

Because information about the bridge's original design and completion hasn't been collected and equipped steel bars in the solid plate of the superstructure are unknown, there lacks relevant information for calculation and checking. According to the survey, the bridge has large amount of current traffic and overloading vehicles over the years of its operation since construction, causing great diseases of the bridge security including: (1)Concrete on the bottom of the solid plate damages and appears vertical and horizontal cracks, which cannot meet the capacity requirements; (2) The main disease of the bridge deck pavement is the serious bridge abutment jump caused by the bridge abutment filler sedimentation and the slab broken. Concrete in the expansion joint breaks, one side of the abutment has no joint and part of the sidewalk has no joint; (3) he front wall and the left wall of No.1 bridge abutment have bad vertical cracks and the right wall has vertical cracks. The cone slope of the bridge abutment appears cracks.

Measure and investigate the bridge, and collect data of the bridge by tools. Draw the bridge map through live inductive date and draw the data by the measuring midvalues. The bridge's general arrangement diagram is as follows.

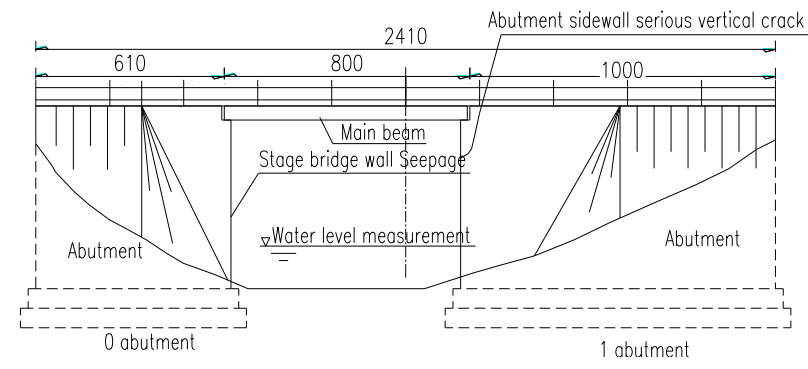

Figure 5. Elevation 


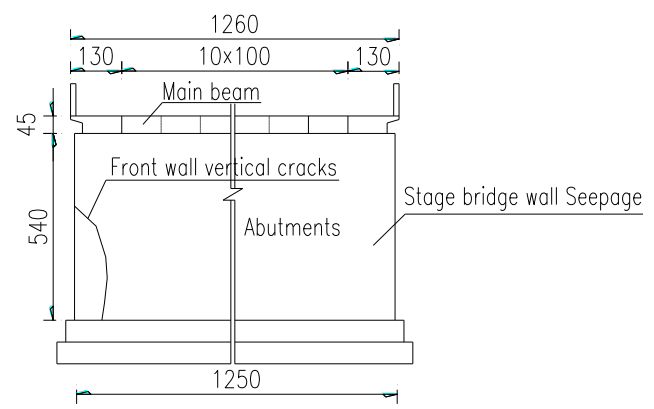

Figure 6. A side view

Due to the traffic volume and overloading, on the bottom of the bridge plate appears some local cracks, and the bearing capacity can't meet the usage requirements. The carbon fiber sheet reinforcing method is chosen considering the small change of the bridge's deflection. Two-layers of carbon fiber sheet with a total width of $300 \mathrm{~mm}$, a total length of $500 \mathrm{~cm}$ are pasted on the bottom of the plate for reinforcing. Measure the bridge data and value all parameters of the bridge. The influence curve of the sensitivity on function $\mathrm{N}$ is shown in diagrams 7 .

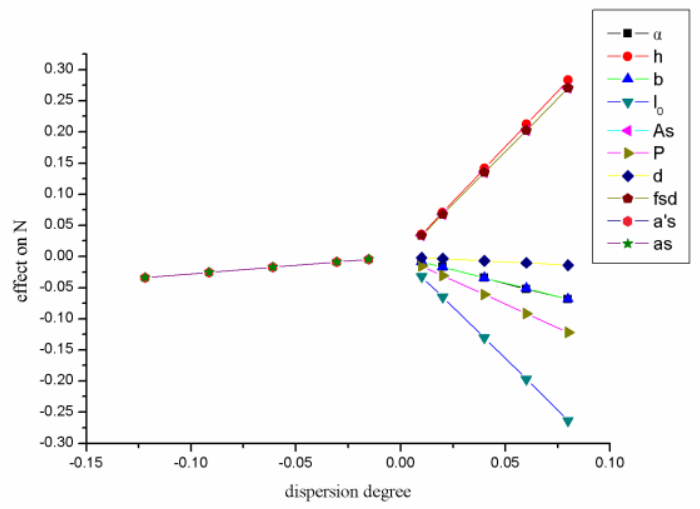

Figure 7. Dispersion diagram of the function function of $\mathrm{N}$ curves

Make error analyses of the magnitude of the effect of function N. Parameter variables which have a great effect on function $\mathrm{N}$ include the solid plate's height $h$, the plate's width $b$, the solid plate's calculated span $l_{0}$, the tensile steel's area $A_{S}$, the steel tensile strength's designed value $f_{s d}$, the concrete's density $\gamma$, the transverse distribution coefficient $\alpha$ and the vehicle load $P$. These variables are sensitive to the function's change, among which those having a greater sensitivity are $l_{0}, h, A_{S}$ and $f_{s d}$.

TABLE I. Three BRIDge EACH PARAMETER VALUe of THE VARIABLE

\begin{tabular}{ccccc}
\hline No. & Factors & Median size & Deviation & variable coefficient \\
\hline 1 & $\mathrm{~h}(\mathrm{~m})$ & 0.4 & 0.04 & 0.1 \\
2 & $\mathrm{l}_{\mathrm{O}}(\mathrm{m})$ & 7.6 & 0.2 & 0.02632 \\
3 & $\mathrm{~A}_{\mathrm{s}}\left(\mathrm{mm}^{2}\right)$ & 3349 & 100 & 0.0299 \\
4 & $\mathrm{f}_{\mathrm{sd}}(\mathrm{MPa})$ & 330 & 20 & 0.061 \\
\hline
\end{tabular}

Sanmen Bridge is a prefabricated solid slab bridge with a single span of $8 \mathrm{~m}$, a simply-supported structure, a simple stress condition. It has functions and can solve the nonprobabilistic reliability index through improving an optimization algorithm. The bridge's compressive zone's height is smaller than $2 a_{s}^{\prime}$ through calculations. The compressive zone's height can be set as $x=2 a_{s}^{\prime}$ according to the specification mandates. Plug $\mathrm{x}$ into pattern (6) and get the following formula:

$$
N=f_{s d} A_{s}\left(h_{0}-a_{s}^{\prime}\right)-0.125 \gamma b(h+d) l_{0}^{2}-0.25 \alpha P l_{0}
$$

According to parameters' sensitivity, determine each parameter's corresponding value or express as corresponding interval and plug it into the formula:

$$
\begin{aligned}
& N=\left(330+20 \delta_{f_{s d}}\right)\left(3349+100 \delta_{A_{s}}\right)\left(0.36+0.04 \delta_{h}\right) \\
& -125 \times\left(25+0.04 \delta_{\gamma}\right)\left(0.99+0.05 \delta_{b}\right)\left(0.5+0.04 \delta_{h}\right) \\
& \left(7.6+0.2 \delta_{l_{0}}\right)^{2}-250 \times\left(300+20 \delta_{p}\right)\left(0.28+0.04 \delta_{\alpha}\right) \\
& \times\left(7.6+0.2 \delta_{l_{0}}\right)
\end{aligned}
$$

According to the results of sensitivity analyses, the analyses of improved one-dimensional optimization algorithm and the positive and negative results of the judgments of all parameters' partial derivatives, determine the value of each parameter variable, calculate the nonprobabilistic reliability index $\eta$ using the matlab as 0.8086 , which is smaller than 1 . We can conclude that the bridge's structure is not reliable and must strengthen it.

\section{B. Evaluating the Reinforcing Effects Based on the One- dimensional Optimization Algorithm}

1) Solving the function after reinforcing

According to data's live measurement gathering, we generalize all parameter variables the function needs, as is shown in the Table II.

TABLE II. AFTER THE IMPACT OF THE CARBON FIBER REINFORCED PARAMETERS

\begin{tabular}{ccccc}
\hline No. & Factors & $\begin{array}{c}\text { Median } \\
\text { Size }\end{array}$ & Deviation & variable coefficient \\
\hline 1 & $\mathrm{E}_{\mathrm{f}}(M p a)$ & 240000 & 10000 & 0.04167 \\
2 & $\mathrm{t}_{\mathrm{f}}(m m)$ & 0.334 & 0.01 & 0.0299 \\
3 & $\mathrm{~b}_{\mathrm{f}}(m m)$ & 300 & 10 & 0.03333 \\
4 & $\varepsilon_{\mathrm{f}}(M p a)$ & 0.007 & 0.0007 & 0.1 \\
\hline
\end{tabular}

The computing methods of sensitivity analysis before reinforcing and after reinforcement are the same. The nonprobabilistic reliability index $\eta$ to be solved is 1.279 , which is bigger than 1. It shows that the bearing capacity of the bridge strengthened by carbon fiber sheet can meet the requirements, so the validity of the reinforcement can be determined. However this computing method aims at the situation that the limit equation is known but many bridges' limit state equation can't be solved easily in the real project 
and it needs the finite element modeling analysis method. Therefore we make modeling numerical solution by preexisting failure point optimization method below, and at the same time, provide ideas and analysis methods to bridges which can't get the function in the carbon fiber sheet reinforcement by comparing with one-dimension optimization algorithm.

2) Evaluating the reinforcement effects based on the failure point optimization method

Use midas/FEA to for modeling. The finite element computing model of the carbon fiber sheet reinforcing the solid plate are shown in diagrams of 8 and 9 .

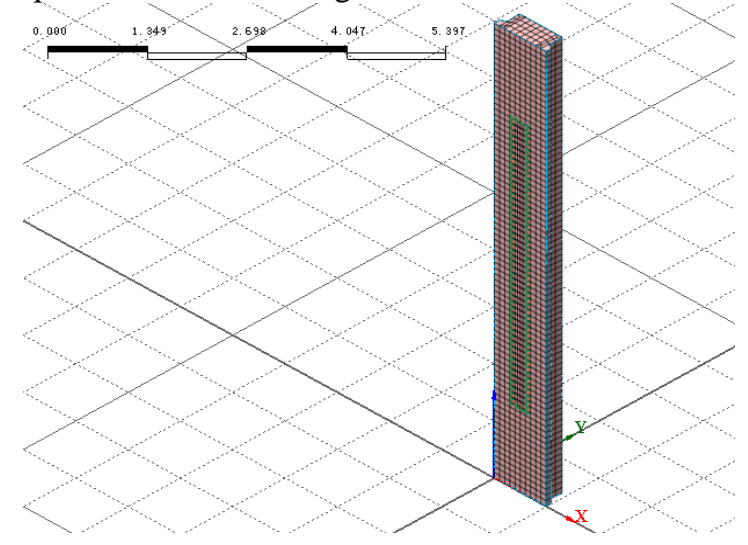

Figure 8. CFRP plane unit grid chart

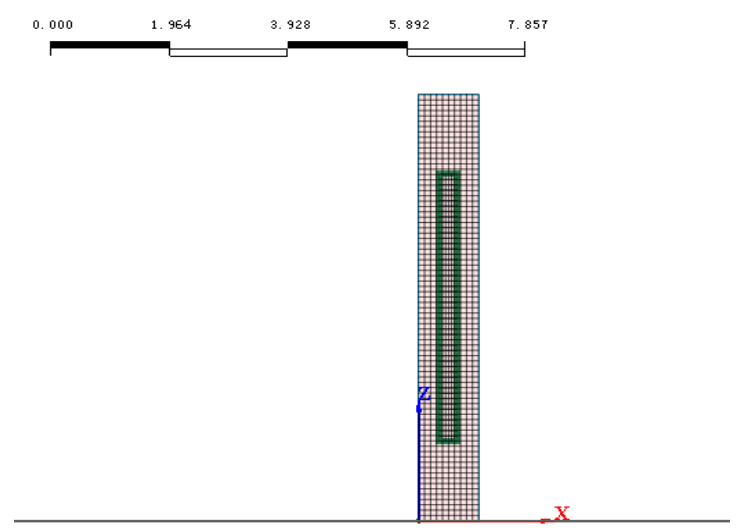

Figure 9. CFRP reinforced solid plate finite element model

Select the mid-span section unit for comparison and analysis, select the internal force combination in the maximum moment state for analysis and make the nonprobabilistic reliability analysis of controlling section when making midas/FEA modeling analyses.

Using the failure point optimization method to solve the non-probabilistic reliability index needs to model and solve the internal force for several times when making the midas modeling analysis. Because of many times of modeling, we analyze evident influence factors including the calculated span- $l_{0}$, the board's height- $h$, the tensile steel's area $-A_{S}$ and the board's elastic modulus $E_{l}$.
TABLE III.

PARAMETER VARIABLES

\begin{tabular}{ccccc}
\hline No & Factors & Size & Deviation & variable coefficient \\
\hline $\mathrm{x}_{1}$ & $\mathrm{l}_{0}$ & 7.6 & 0.2 & 0.02632 \\
$\mathrm{x}_{2}$ & $\mathrm{~h}$ & 0.4 & 0.04 & 0.1 \\
$\mathrm{x}_{3}$ & $\mathrm{E}_{1}$ & 34600 & 2000 & 0.0578 \\
$\mathrm{x}_{4}$ & $\mathrm{~A}_{\mathrm{s}}$ & 3349 & 100 & 0.0299 \\
\hline
\end{tabular}

1. Determine M's increase related to each variable: we know by analyzing that $x_{1}, x_{2}, x_{4}$ are increasing functions of $\mathrm{M}$ and that $x_{3}$ is a decreasing function of $\mathrm{M}$, then $x_{i}^{*}=x_{i}^{c}-x_{i}^{r} \eta^{k}(\mathrm{i}=1,2,4), x_{3}^{*}=x_{3}^{c}+x_{3}^{r} \eta^{k}$.

2. Select the original point. Setting $\eta^{1}=0$, we can get the mid-value failure point and get $M^{1}$ by using the finite element software to compute internal force. Set the original point $\eta^{1}=1$, plug the above confirmed new failure point $x_{i}$ into the software Midas/FEA and get the calculated result related to $M^{1}$ as is shown in the table below:

TABLE IV. POINT OF FAILURE TO FIND THE OPTIMAL METHOD OF CALCULATION RESUlTS

\begin{tabular}{ccccccc}
\hline No & $\eta *$ & $x_{1}$ & $x_{2}$ & $x_{3}$ & $x_{4}$ & $M$ \\
\hline 1 & 1 & 7.8 & 0.36 & 32600 & 3249 & 86 \\
2 & 2 & 8.2 & 0.28 & 28600 & 3049 & -34 \\
3 & 1.717 & 8.1 & 0.29 & 29166 & 3077 & -24 \\
4 & 1.037 & 8.0 & 0.32 & 30352 & 3145 & 7 \\
5 & 1.19 & 8.0 & 0.31 & 30220 & 3130 & 3 \\
6 & 1.305 & 8.1 & 0.31 & 29990 & 3119 & 0.4 \\
7 & 1.323 & 8.1 & 0.31 & 29954 & 3117 & 0.2 \\
\hline
\end{tabular}

The function is convergent when the number of iterations is 7. So the non-probabilistic index is 1.323. Conclusion: the result of non-probabilistic parameters calculated by the failure point optimization method is bigger than the result by the one-dimension optimization algorithm. There is a certain error between them. The error is $3 \%$ according to the computing results of them, which validates the validity of the analysis of reinforcing bridge with carbon fiber sheet by the failure point optimization method and provides supports to complicated strengthened bridge's non-probabilistic reliability computing.

\section{Optimization research of the width and layer amount of carbon fiber sheet reinforcing}

We discuss the influence rules of non-probabilistic reliability index after reinforcing by changing the amount of carbon fiber sheet used in reinforcing. The carbon fiber sheet parameters include the paste thickness $b_{f}$ and the carbon fiber sheet paste layer $\eta_{f}$. We solve the computing results of the non-probabilistic reliability index according the two adapted parameters variables when reinforcing. 
TABLE V.CFRP LAYERS AFFECT CHANGE ON A NON-PROBABILISTIC RELIABILITY INDEX

\begin{tabular}{ccc}
\hline Width & Layers changes & index \\
\hline 300 & From one to two & 0.158514493 \\
300 & From two to three & 0.123534011 \\
400 & From one to two & 0.231793961 \\
400 & From two to three & 0.143475126 \\
500 & From one to two & 0.220672683 \\
500 & From two to three & 0.157930108 \\
600 & From one to two & 0.244304792 \\
600 & From two to three & 0.167929293 \\
\hline
\end{tabular}

Through comparison analysis above we can conclude that the effect of paste layer's change on carbon fiber sheet reinforcement's non-probabilistic reliability index is great. Because the non-probabilistic reliability index's increase from one-layer to two-layer is bigger than the change from two-layer to three-layer, we choose to paste two-layer often. The effect of width on non-probabilistic reliability index is smaller compared with the layer. So the bridge's paste width is $300 \mathrm{~mm}$ and the layer is two. The non-probabilistic reliability index is 1.279 which meets the requirements.

\section{THE REINFORCING SCHEME}

Because there are some local cracks on the bottom of the bridge and the bearing capacity can't meet the requirements, two layers of first-class carbon fiber sheet are pasted on the bottom of the plate with a total width of $300 \mathrm{~cm}$ and a total length of $500 \mathrm{~cm}$ for reinforcing. The specific construction process is shown in Fig. 10

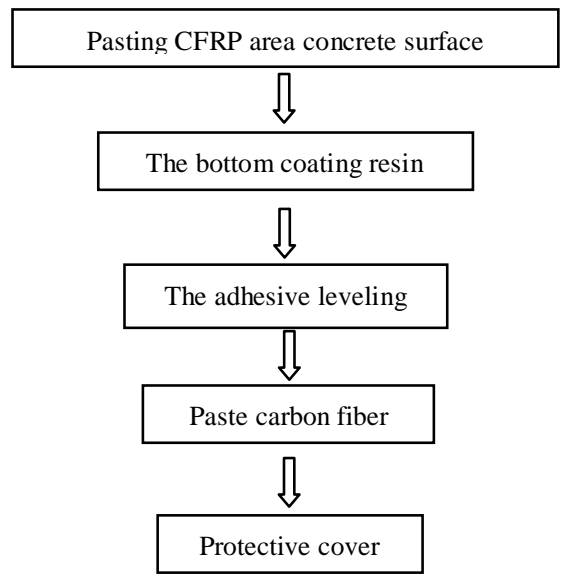

Figure 10. Flowchart of the reinforcing construction of carbon fiber sheet

All cracks in the side wall and the front wall of the bridge abutment with width greater than 0.1 have grouting treatment. The grouting glue adapts epoxy resin and the front wall and the side wall of the bridge abutment are reinforced by outsourced reinforced concrete.

New bridge deck pavement should be designed and the drainage system should be improved for damaged roads and rails to extend the life of the bridge and improve its appearance. A C40 waterproof concrete layer with $10 \mathrm{~cm}$ thick is re-paved on the bridge, which adapts single-layer rebar and has a double cross slope of $2 \%$. Replace the rail into marble rail. Reconstruct the deck drainage system made of PVC pipes with a layout spacing of $5 \mathrm{~m}$ and an amount of 6 in total. Dismantle the origin expansion joints of the deck and rebuild 2 GQF-C-40 expansion joints.

\section{CONCLUSIONS}

We apply the non-probabilistic reliability theory to this field for the first time by the research on reinforcing bridge with carbon fiber sheet and offer new research methods and approaches for reinforcing bridge with carbon fiber sheet. The main research results are listed as follows:

(1).Apply the non-probabilistic reliability theory to reinforcing bridge with carbon fiber sheet for the first time and offer the determining methods of the function;

(2).Apply the existing failure point optimization to build Midas/FEA model and get numerical solutions, and at the same time, offer new thoughts and analysis methods for bridges with different functions by the paste carbon fiber sheet reinforcing through comparing with the improved onedimensional algorithm;

(3).Point out that the change of the paste layer amount has much effect on the non-probabilistic reliability index of the carbon fiber sheet reinforcing. The increase of the nonprobabilistic reliability index from pasting one layer to two layers is larger than that from pasting two layers to three layers. Introduce the process of reliability assessment and reinforcement scheme selection, and verify the validity of methods in this paper.

\section{ACKNOWLEDGEMENTS}

This work is financially supported by National Natural Science Foundation of China (51408444)

\section{REFERENCE}

[1] Chen hong-mei,Damage analysis of reinforced concrete bridge and its maintenance and reinforcement[D], dalian,2011

[2] Zhao liang,Study on the application of prestressed carbon fiber sheet in the reinforcement of PC plate girder bridge[D], Xi'an,2013

[3] Kaiser.H. Bewehren von stahlbeton mit kohlenstoffaserverstarken Epoxidharzen[D].Federal Technical Highschool of Zurich,1989

[4] Deuring.M. Verstarken von stahlbeton mit gespannten faserver bundwerkstoffen[R]. Swiss Federal Laboeatories for Material Testing and Rearch,1993

[5] Meier.U,Deuring.M. Fiber-Reinforced-Plastic(FRP)Reinforcement for Concrete Structures[J]. Properties and Application1993,pp:423434

[6] U.Meier. Bridge repair with high performance composite materials[J]. Material and Technique,1987(4),pp:125-94

[7] Wu li-li.Yu da-chuan, Application of FRP materials in structural reinforcement and the characteristics of FRP and its application in Civil Engineering. $[\mathrm{J}]$, journal of harbin university of civil engineering and architecture2000.33(6),pp: 26-30

[8] ACI Committee 440.Guide for the Design and Construction of Bonded FRP System For Strengthening Concrete Structures[S]. Detroit: American Concrete Institute,2000

[9] Qiuji Wei, Zhang Ruijun, Cong Dongsheng mechanical parts reliability design theory and methods of Engineering Design[J], 2014 (8),pp:401-406 
[10] .Jiang tao, Chen jian-jun, Zhang jian-guo,et al. Response surface method based on interval models and interval analysis[J]. Machine Design and Research, 2005, 21(6), pp:12-16

[11] Chen xu-yong, Fan Jian-ping. An improved one-dimensional optimization algorithm in non-probabilistic reliability investigation and its application in bridge assessment [J]. Engineering Mechanics, 2011,28(5),pp:21-25
[12] Chen xu-yong, Fan Jian-ping. Application of convex model based on non-probabilistic concept of reliability in bridge assessment. J. Huazhong 